\title{
Simulation of photoelectron emission from metallic nanoparticles under laser irradiation ${ }^{\star}$
}

\author{
Laszlo Budai ${ }^{1}$, Zsuzsanna Márton $^{2,3}$, Péter Dombi ${ }^{3,4}$, and Károly Tőkési ${ }^{1,3, a}$ \\ 1 Institute for Nuclear Research, Hungarian Academy of Sciences (Atomki), 4026 Debrecen Bem tér 18/c, Hungary \\ 2 Institute of Physics, University of Pécs, Pécs, Hungary \\ 3 ELI-ALPS, ELI-HU Non-profit Ltd., Dugonics tér 13, 6720 Szeged, Hungary \\ 4 Wigner Research Centre for Physics, Budapest, Hungary
}

Received 13 December 2018

Published online 4 July 2019

(C) The Author(s) 2019. This article is published with open access at Springerlink.com

\begin{abstract}
We developed a new computer simulation code that calculates trajectories of photoelectrons emitted from nanoparticles by laser excitation. The code uses the pre-calculated electric field obtained by finite-difference time-domain simulations as input. The photoelectron trajectories emitted from silver nanoparticles were calculated using the classical trajectory Monte Carlo method, where the image force towards the surface is taken into account. We show that our present code is suitable to describe the recent experimental findings reasonably well. Significant effect of the image acceleration to the calculated electron spectra was observed. We found that the calculated energy distributions of photoelectrons are in agreement with the recent experiments.
\end{abstract}

\section{Introduction}

The photoelectric effect was first explained by Einstein [1] more than a hundred years ago. A number of devices use this effect, for example photocells, photodiodes and solar cells. Recently it has become an indispensable part of our everyday life. Attosecond physics is a new and rapidly developing field driven by recent advances in laser technology. Attosecond science holds the promise to observe and to control the optical-field driven electronics working at clock speeds six orders of magnitude faster than current conventional electronics. Combining this extremely small time scale with nano-dimensional materials gives us fairly new techniques for new discoveries.

One of the results of this recognition is that electron emission from nanoparticles induced by laser excitation have been raising great interest recently $[2,3]$. When the incident electric field (light) interacts with a metal nanoparticle, it can generate collective oscillation of the conduction electrons. This collective oscillation is known as localized surface plasmon. Based on this new investigation a new research field for exploiting of the resonant interaction is established and it is called plasmonics. Besides focusing on challenging fundamental and basic science both in experimental and theoretical aspects, plasmonics promises many technical applications.

\footnotetext{
* Contribution to the Topical Issue "Many Particle Spectroscopy of Atoms, Molecules, Clusters and Surfaces (2018)", edited by Károly Tőkési, Béla Paripás, Gábor Pszota, and Andrey V. Solov'yov.

${ }^{a}$ e-mail: tokesi@mta.atomki.mta.hu
}

To name just a few, we can mention plasmonic biosensors $[4,5]$, optoelectronics [6,7], photovoltaics [8] and construction of ultrafast nanoemitters [9-13].

Along this line, we developed a computer simulation code based on the classical dynamics to mimic the electron emission from a periodic nanostructure. First, the time dependent electric field was calculated. Kretschmann configuration [14] was considered for plasmonic field generation. For calculating the electric field in the vicinity of the surface, we used the finite-difference time-domain (FDTD) simulations [15]. The trajectories of the spontaneously emitted electrons were then followed in the calculated time dependent electromagnetic field. The electron trajectories were calculated both with and without the image force between the emitted electron and its mirror charge. Atomic units are used throughout the paper unless otherwise indicated.

In this work we show that our present code is suitable to describe the recent experimental findings with reasonably well. The calculated energy distributions of photoelectrons are compared with the experimentally measured distributions.

\section{Plasmonic field}

The plasmonic field was generated in Kretschmann configuration [14]. The modelled setup consisted of bulk fused silica covered by a $48 \mathrm{~nm}$, atomically flat silver film with a single semi-ellipsoidal protrusion with axes of $2 \mathrm{~nm}, 15 \mathrm{~nm}$, $15 \mathrm{~nm}$. The incident laser pulse was an $800 \mathrm{~nm}, 5$ fs plane wave. The electric field of our laser pulse is described 
by a simple plane wave modulated with a Gaussian envelop as:

$$
E(t)=\sin \left\{-2 \pi f\left(t-t_{0}\right)\right\} \exp \left\{-\left(t-t_{0}\right)^{2} / \tau^{2}\right\}
$$

where $f=374.74 \mathrm{THz}, t_{0}=100 \mathrm{fs}$ and $\tau=4.55 \mathrm{fs}$.

The angle of incidence was $44.6^{\circ}$. Recently the FDTD simulation technique is widely used for describing the have been used to describe the near and the far-field optical responses of disordered metal-dielectric composites [16-19]. The FDTD method or Yee algorithm $[20,21]$ is a well-known technique based on space and time discretization of the Maxwell curl equations. The dimensions of the 2D simulation space were $20 \mathrm{~nm}$ by $60 \mathrm{~nm}$, with $0.5 \mathrm{~nm}$ spatial resolution, and the considered time window was 50 fs with 30.5 as time resolution.

Figure 1 shows the calculated electric field components in the vicinity of a semi-ellipsoid protrusion of an $\mathrm{Ag}$ surface layer at different instants.

\section{Trajectory simulation}

The time dependent electric field (see Fig. 1) generated by FDTD simulations was the input data for our trajectory calculations. Each line of the input file contains, the time $(\mathrm{t}[\mathrm{fs}])$ the spatial coordinates (x; $\mathrm{y}[\mathrm{nm}])$ and the electric field components in $\mathrm{V} / \mathrm{m}$. As the input data file is a result of a sampling process, we have $\mathrm{K}$ discrete time slices, and a discrete electric field (spatial grid) is assigned to each time slice. This means we need to interpolate between the spatial coordinates, and time is also interpolated. A typical input file is $503 \mathrm{MB}$ and contains 6 million lines of data. The electric fields are ordered by time, and the sampling points form an equidistant grid. This means that the lines are ordered by time, then by $\mathrm{x}$ and by y. Complexity of loading the data is proportional to $\mathrm{O}(\mathrm{n})$, linear. Complexity of accessing the data is proportional to $\mathrm{O}(\log (\mathrm{n}))$. The simulations were performed in atomic units.

During the simulation we need to interpolate between the time slices. As a result, we generate an interpolated electric field to the given time point, then we locate the neighbourhood of the electron and calculate the corresponding electric field. The schematic diagram of the applied method can be seen in Figure 2. The classical equations of motion were solved numerically by applying the standard Runge-Kutta method. During the simulation we took into account the image force $\left(F_{i}\right)$ perpendicular to the surface according to the form:

$$
F_{i}=q^{2} /\left(4 d^{2}\right),
$$

where $d$ is the distance between the charge $q$ and the surface. In our code it is straightforward to switch the classical image force on and off. We performed simulations in both modes.

\section{Results and discussion}

We performed a large number of classical trajectory simulations based on our new code for the study of the photoelectron emission from the semi-ellipsoid protrusion of an
Ag surface layer after laser irradiation. For each simulation we follow 20 million trajectory histories. The initial positions of emitted photoelectrons are selected randomly on the semi-ellipsoid protrusion surface of Ag. The escaping energy was close to zero. The initial angle of the electrons is taken randomly from the uniform distribution. First, we analyzed the individual trajectories in the vicinity of the silver nano-hill. The typical electron trajectories are shown in Figures 3 and 4 . When the image force is not taken into account, most of the electron trajectories move away from the surface in the time dependent electric field (see Fig. 3).

Taken into account the image acceleration toward the Ag surface, however, we can identify a large number of trajectories returning to the surface (traj4 in Fig. 4). The trajectory type 4 is a typical path when the electron returns to the surface. These electrons can either penetrate into the Ag material doing again random walk in the vicinity of the surface and in the bulk, or can be elastically backscattered directly. If the electrons penetrate into the surface we may lose them because they may not be able to escape again from the surface.

Figure 5 shows the energy distribution of photoelectrons ejected from the silver nano-protrusion after laser irradiation at $230 \mathrm{GW} / \mathrm{cm}^{2}$ focused beam intensity. Figure $5 \mathrm{a}$ shows the experimental data taken from [22]. They found a double peak structure energy distribution. Our recent simulation results are shown in Figure 5b where the image acceleration is not taken into account, and in Figure 5c where the classical image force toward the Ag surface was taken into account. We found significant differences between the two distributions. Without image force, the obtained electron energies are smaller than with it. In Figure 5b, the energy distribution is concentrated close to the 0 energy and the maximum energy of the photoelectrons is around $19 \mathrm{eV}$.

In contrast, when the image acceleration is taken into account we found much wider energy distributions with higher energies for the photoelectrons. The experimentally obtained double peak structure is also obtained.

Figure 6 shows the comparison between the experimentally measured energy distributions of photoelectrons ejected from a nanostructured silver surface after laser irradiation at $230 \mathrm{GW} / \mathrm{cm}^{2}$ focused beam intensity and the result of our Monte Carlo simulation. We normalized these two peaks in such a way that their asymptotic behaviour is the same at higher energies. Our theoretical curve is in agreement with the experimental observations. We mimic the double peak structure of the energy distribution. The agreement even in absolute scale is also reasonable. We note that the Monte Carlo simulation gives higher intensities for the case of the first peak

We analyzed further the electron trajectories searching for the possible reason of the double peak structure. As we stated before, trajectories when the electrons return to the surface are obtained frequently when image acceleration is taken into account in the simulation (traj4 in Fig. 4). Figure 7 shows the electron trajectory first return back to the surface and later elastically backscattered again from the surface. In this case the electron can travel two times in the time dependent electric field and may gain more 

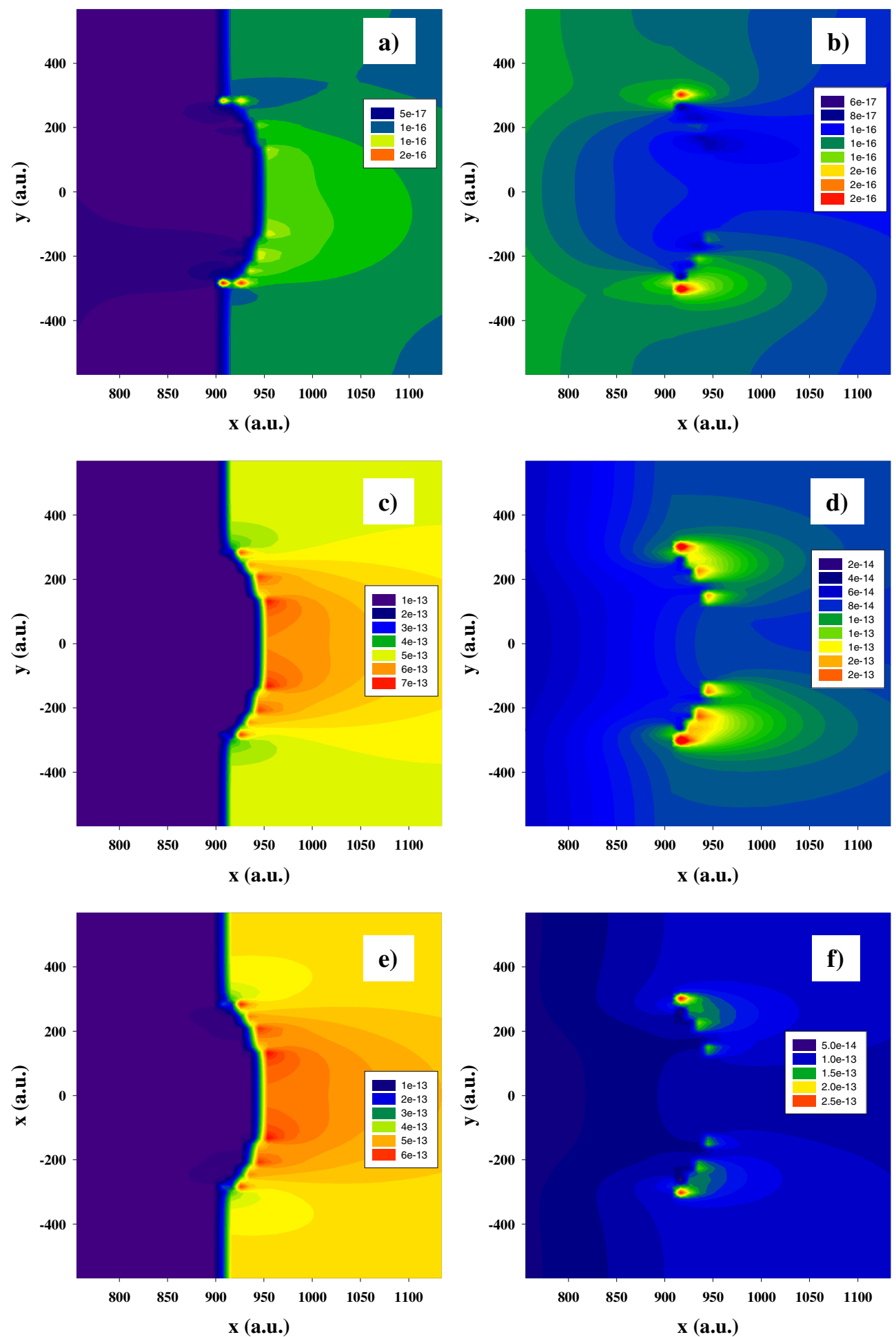

Fig. 1. The $E_{x}(\mathrm{a}, \mathrm{c}, \mathrm{e})$ and $E_{y}(\mathrm{~b}, \mathrm{~d}, \mathrm{f})$ components of the electric field $\mathrm{E}$ in vacuum, in the vicinity of a semi-ellipsoid protrusion of an $\mathrm{Ag}$ surface layer, illuminated by an oblique incident, plane fs pulse through a transparent prism, in Kretschmann configuration. (a) $E_{x}$ at $t=90 \mathrm{fs}$, (b) $E_{y}$ at $t=90 \mathrm{fs}$, (c) $E_{x}$ at $t=110 \mathrm{fs}$, (d) $E_{y}$ at $t=110 \mathrm{fs}$, (e) $E_{x}$ at $t=130 \mathrm{fs}$, (f) $E_{y}$ at $t=130 \mathrm{fs}$. 


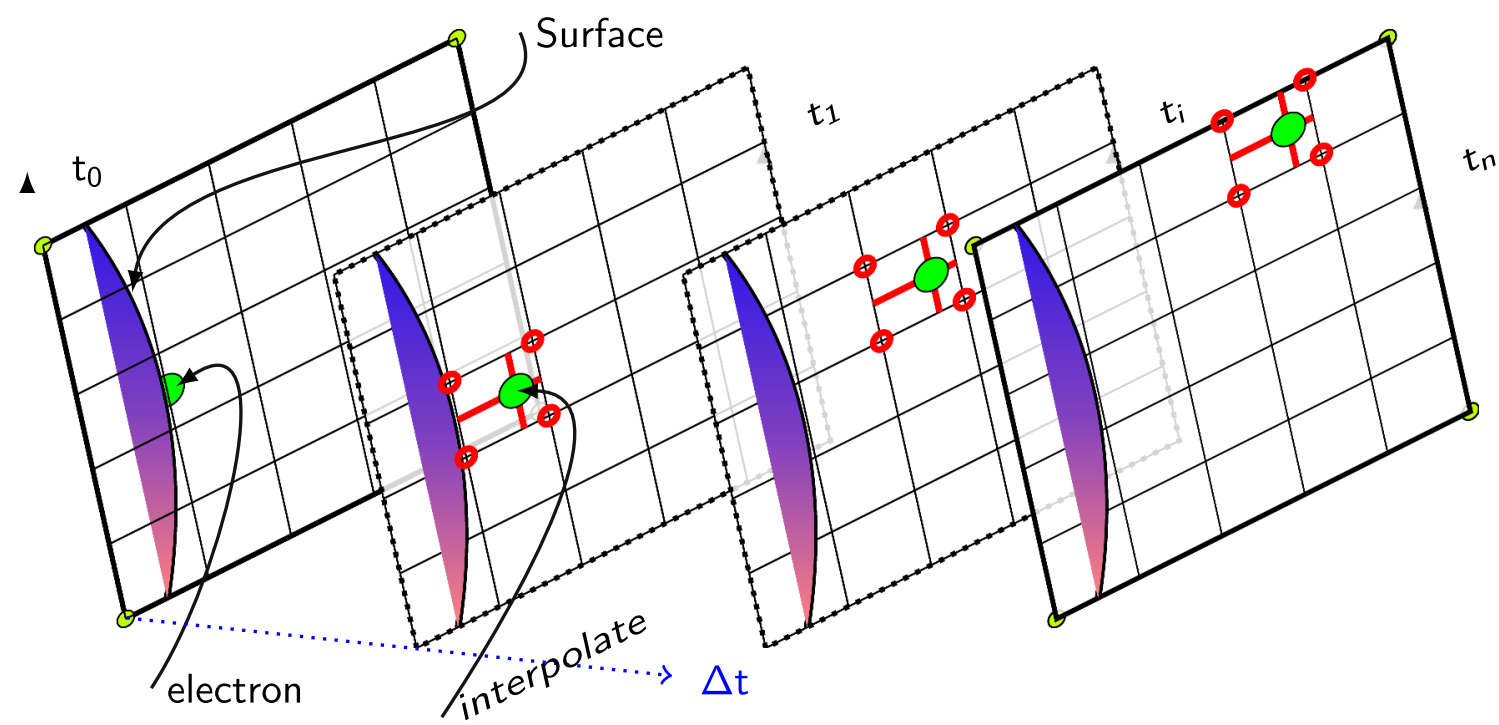

Fig. 2. Schematic diagram of the calculation procedure.

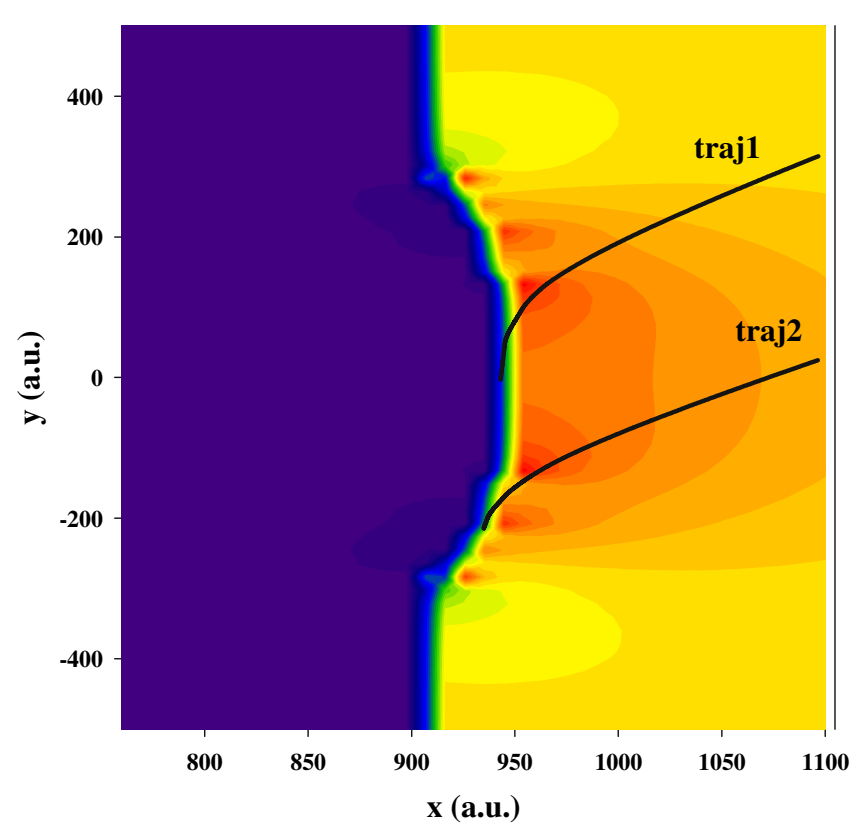

Fig. 3. Typical electron trajectories calculated without the image acceleration toward the surface in the vicinity of the silver semi-ellipsoid after laser irradiation.

energy compared to the case when it travels only once (traj3 in Fig. 4).

For the clear identification, we plan further experimental and theoretical work, which is in progress.

\section{Conclusion}

A new computer simulation code was developed, which calculates the trajectories of photoelectrons emitted from laser-excited nanometer scale semi-ellipsoid surface protrusion. The code uses as input the pre-calculated electric

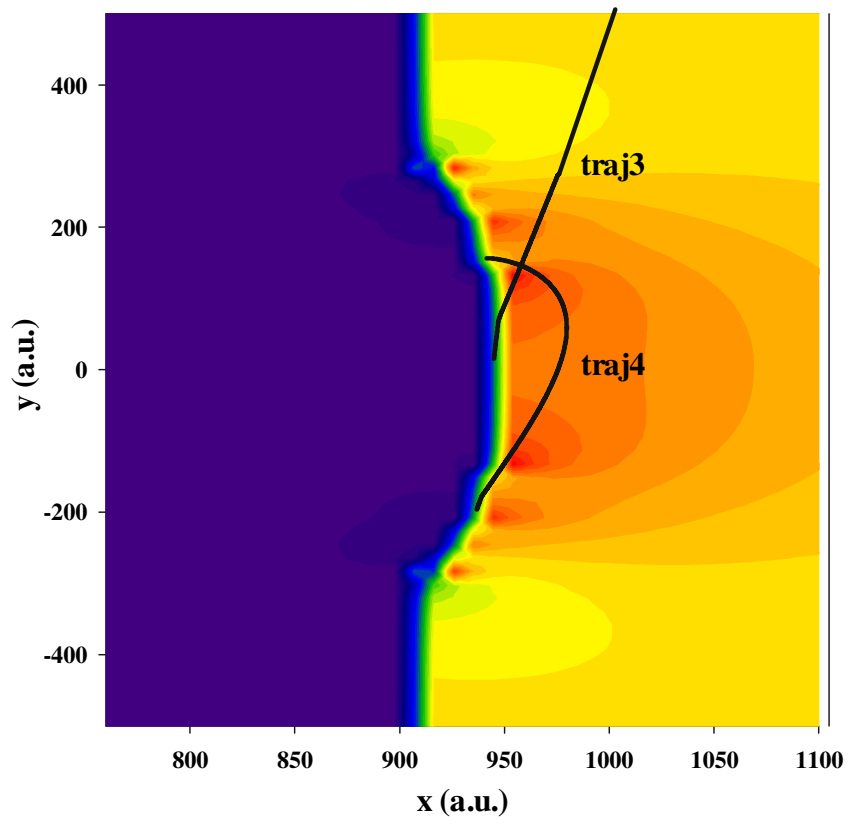

Fig. 4. Typical electron trajectories calculated with the image acceleration taken into account in the vicinity of the silver semi-ellipsoid after laser irradiation.

field obtained by the finite-difference time-domain simulations. We have shown that our present code is suitable to describe recent experimental findings reasonably well. We found that the image acceleration toward the surface plays a dominant role in shaping the electron trajectories. We found that the calculated energy distributions of photoelectrons are in agreement with the recent experiments. The experimentally obtained double peak structure was identified in our calculated spectra. Electrons returning to the surface and scattered on it again may gain higher final energies, and they contribute to the second peak at around $21.5 \mathrm{eV}$. On the other hand, electrons following 


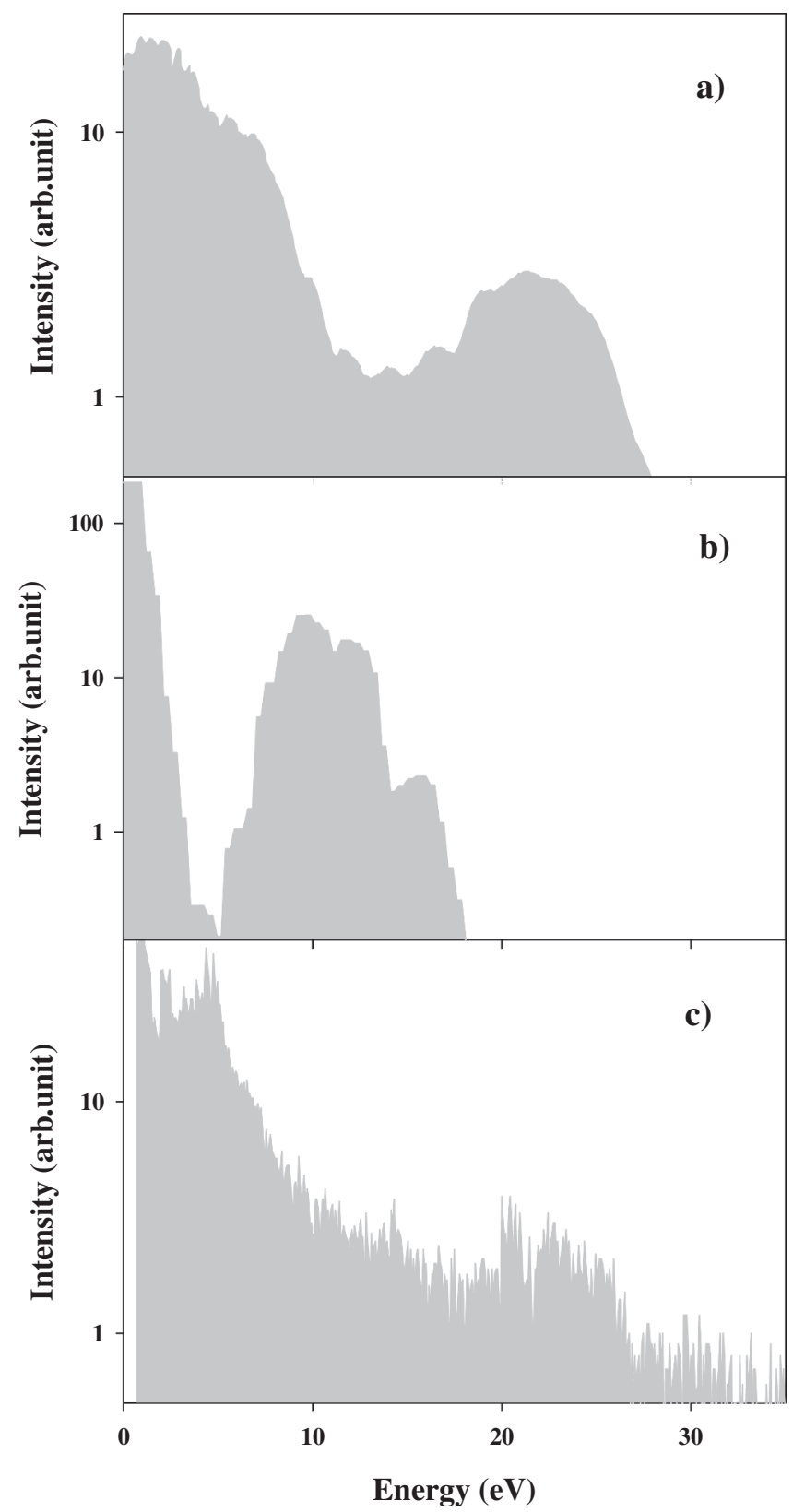

Fig. 5. Energy distribution of photoelectrons ejected from silver nanoparticles after laser irradiation at $230 \mathrm{GW} / \mathrm{cm}^{2}$ focused beam intensity. (a) Experimental data taken from [22]. (b) Simulation without the image acceleration toward the surface. (c) Simulation with image acceleration toward the surface taken into account.

a pathway without returning to the surface mostly will increase the peak at a lower energy. Further tests are in progress for justifying this explanation of the double-peak structure of the electron energy spectra.

Open access funding provided by Institute for Nuclear Research. The work was supported by the National Research, Development and Innovation Office (NKFIH) Grant KH126886. Partial support by the ELI-ALPS project is also acknowledged. The ELI-ALPS project (GOP-1.1.1-12/B-2012-

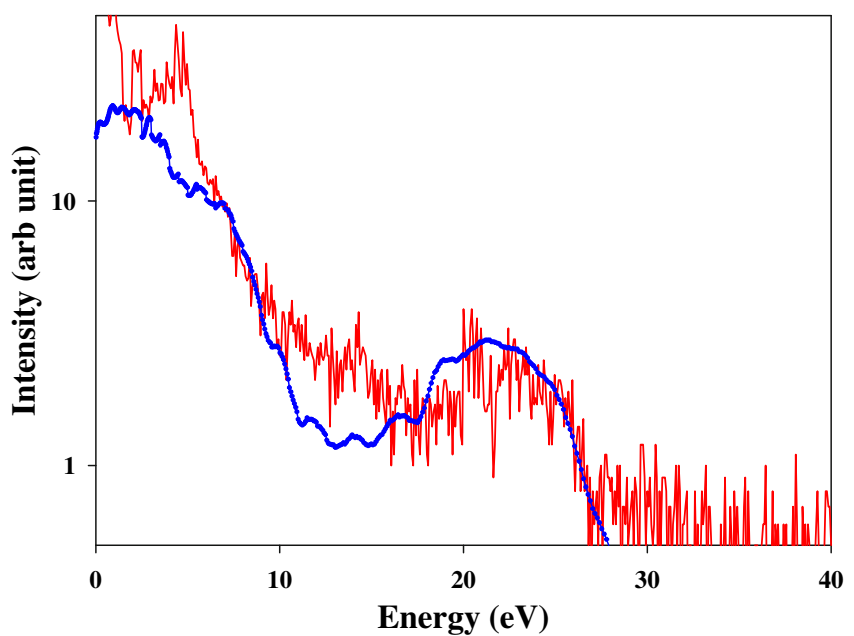

Fig. 6. Comparison of the energy distribution of photoelectrons ejected from silver nanoparticles after laser irradiation at $230 \mathrm{GW} / \mathrm{cm}^{2}$ focused beam intensity. Result of Monte Carlo simulation (thin solid line, red in color), the experimental data from reference [22] (thick solid line, blue in color).

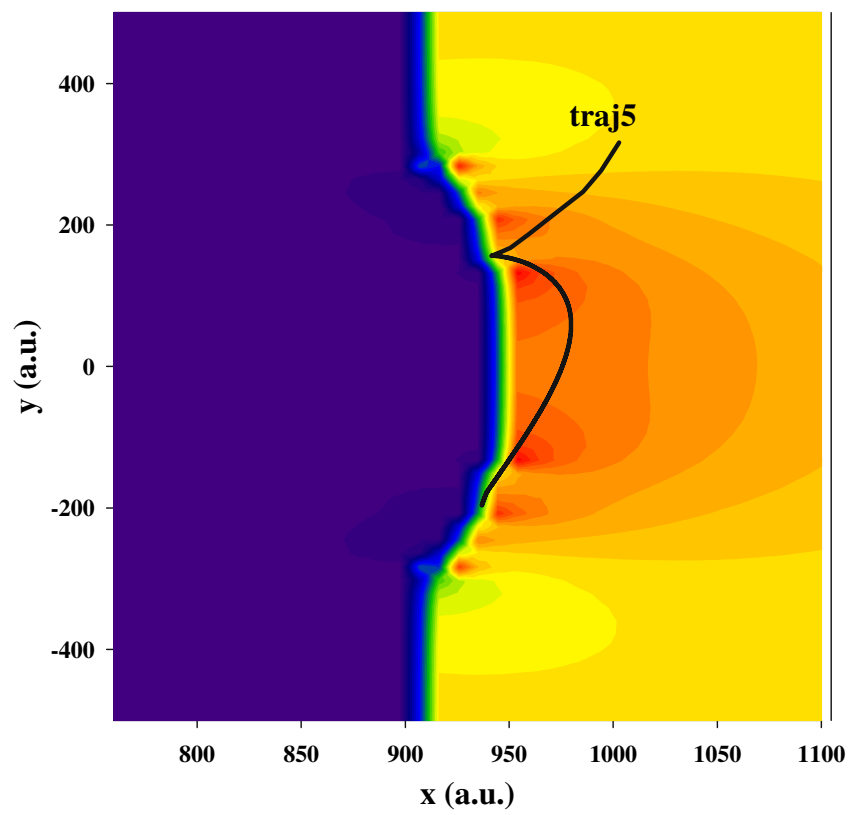

Fig. 7. Typical backscattered electron trajectory in the vicinity of the silver nano-ellipsoid after laser irradiation with image force taken into account.

000, GINOP-2.3.6-15-2015-00001) is supported by the European Union and cofinanced by the European Regional Development Fund. We also acknowledge support from the Max Planck Society in the form of a Partner Group Grant.

\section{Author contribution statement}

All authors discussed the results and contributed to the final manuscript. L. Budai: development of Monte Carlo simulation code, performance of the trajectory 
calculations, preparing the figures of the manuscript, $\mathrm{Z}$. Márton: performance of the finite-difference time-domain simulations, P. Dombi: definition of the model and analysis/interpretation of the simulation results, K. Tőkési: adviser of the development of simulation code, preparing the figures of the manuscript.

Open Access This is an open access article distributed under the terms of the Creative Commons Attribution License (http://creativecommons.org/licenses/by/4.0/), which permits unrestricted use, distribution, and reproduction in any medium, provided the original work is properly cited.

\section{References}

1. A. Einstein, Ann. Phys. (Berlin) 17, 132 (1905)

2. P. Dombi, A. Hörl, P. Rácz, I. Márton, A. Trügler, J.R. Krenn, U. Hohenester, Nano Lett. 13, 674 (2013)

3. P. Rácz, Zs. Pápa, I. Márton, J. Budai, P. Wróbel, T. Stefaniuk, C. Prietl, J.R. Krenn, P. Dombi, Nano Lett. 17, 1181 (2017)

4. J.N. Anker, W.P. Hall, O. Lyandres, N.C. Shah, J. Zhao, R.P. Van Duyne, Nat. Mater. 7, 442 (2008)

5. J. Homola, Chem. Rev. 108, 462 (2008)

6. A. Satharasinghe, T. Hughes-Riley, T. Dias, Sci. Rep. 8, 16205 (2018)

7. R. Hao, Z. Ye, Y.J. Gu, X. Peng, H. Chen, E. Li, Sci. Rep. 8, $16830(2018)$
8. A. Luque, S. Hegedus, eds., Handbook of Photovoltaic Science and Engineering (John Wiley \& Sons Ltd, 2003)

9. V. Giannini, A.I. Fernández-Domínguez, S.C. Heck, S.A. Maier, Chem. Rev. 111, 3888 (2011)

10. M. Fujiwara, K. Toubaru, T. Noda, H.-Q. Zhao, S. Takeuchi, Nano Lett. 11, 4362 (2011)

11. C. Karnetzky, P. Zimmermann, C. Trummer, C.D. Sierra, M. Wörle, R. Kienberger, A. Holleitner, Nat. Commun. 9, 2471 (2018)

12. J. Vogelsang, J. Robin, B.J. Nagy, P. Dombi, D. Rosenkranz, M. Schiek, P. Groß, C. Lienau, Nano Lett. 15, 4685 (2015)

13. G. Herink, D.R. Solli, M. Gulde, C. Ropers, Nature 483, $190(2012)$

14. E. Kretschmann, H. Raether, Z. Naturforsch. A 23, 2135 (1968)

15. Lumerical FDTD Solutions, https://www.lumerical. com/tcad-products/fdtd/

16. S. Buil, J. Laverdant, B. Berini, P. Maso, J.-P. Hermier, X. Quélin, Opt. Express 20, 11974 (2012)

17. Y. Liu, R. Cheng, L. Liao, H. Zhou, J. Bai, G. Liu, L. Liu, Y. Huang, X. Duan, Nat. Commun. 2, 579 (2011)

18. N. Okada, J.B. Cole, Micromachines 3, 168 (2012)

19. O. Sofiane, S. Ouaskit, Am. J. Electromagn. Appl. 5, 14 (2017)

20. K.S. Yee, IEEE Trans. Antennas Propag. 14, 302 (1966)

21. A. Taflove, S.C. Hagness, Computational Electrodynamics: The Finite-Difference Time-Domain Method (Artech, 2005)

22. J. Budai, Z. Pápa, I. Márton, P. Wróbel, T. Stefaniuk, Z. Márton, P. Rácz, P. Dombi, Nanoscale 10, 16261 (2018) 